\title{
Dosimetric Comparison of Pelvic Bone Marrow Preservation between Volumetric-Modulated Arc Therapy and Intensity-Modulated Radiotherapy in Radical Radiotherapy of High-Risk Prostate Cancer
}

\author{
Ehab Saad $^{1,2}$, Khaled Elshahat ${ }^{2,3}$, Hussein Metwally ${ }^{2,4}$ \\ ${ }^{1}$ Department of Clinical Oncology and Nuclear Medicine, Kasr Al-Ainy School of Medicine, Cairo \\ University, Cairo, Egypt; ${ }^{2}$ Department of Radiation Oncology, Dar Al-Fouad Hospital, Giza, Egypt; ${ }^{3}$ \\ Department of Clinical Oncology, Al-Azhar University, Cairo, Egypt; ${ }^{4}$ Department of Clinical \\ Oncology, Fayoum University, Fayoum, Egypt
}

Background: Pelvic bone marrow (PBM) preservation is one of the factors that should be taken into consideration while choosing a technique for radiotherapy of pelvic malignancies.

Aim: To dosimetrically compare between volumetric-modulated arc therapy (VMAT) and intensity-modulated radiotherapy (IMRT) in PBM preservation in radical treatment of high-risk prostate cancer.

Methods: In 26 patients with high-risk prostatic carcinoma, dual arc VMAT and 7 fields IMRT plans were generated. In every patient, two targets were defined, clinical target volume (CTV) including the prostate and seminal vesicles (CTVPSV) and CTV including pelvic lymph nodes (CTV-LN). The organs at risk delineated were the rectum, urinary bladder, small intestine, bulb of the penis, femoral heads bilaterally and PBM. The dose prescribed to the CTV-PSV was 76 Gy in 38 fractions given over 7.5 weeks and the dose to CTV-LN was 54 Gy in 38 fractions given over 7.5 weeks. Planning target volume (PTV) was created from the CTV with a margin of $5 \mathrm{~mm}$ in all direction. For assessment of PBM dose, V10, V20, V30, V40, V50 and mean dose were calculated. The dose volume histogram of PTV and PBM for both techniques was compared.

Results: The mean dose of PTV 54 Gy was achieved in both techniques adequately with better sparing of organs at risk with the VAMT technique. The mean dose for PBM in the VMAT technique was significantly less than that in the IMRT (21.7 Gy vs. $25.8 \mathrm{~Gy}$, respectively; $\mathrm{p}<0.001)$. The significant differences in PBM doses were in the range of $20 \mathrm{~Gy}$ to 40 Gy.

Conclusion: In radical treatment of prostate cancer, VMAT technique can offer comparable conformality to IMRT with better PBM preservation. Awareness of PBM delineation and reduction of its doses using VMAT can help to decrease the hematological toxicity.

Keywords: Prostate cancer, Radiotherapy, IMRT, VMAT, Pelvic bone marrow, Bone marrow sparing

Corresponding author: Ehab Saad, MD; Department of Clinical Oncology and Nuclear Medicine, Kasr Al-Ainy School of Medicine, Cairo University, Cairo, Egypt; Email: ehab.saad239@ gmail.com

Submitted: 2-May-2019, Revised: 2-June-2019, Accepted: 5-June-2019, Published online: 6-June-2019

(cc) BY

\section{INTRODUCTION}

Prostate cancer is considered the most common malignancy in males. It is the $4^{\text {th }}$ leading reason of cancer-related death in the developed countries ${ }^{1}$. Prostatic radiation therapy concurrent with androgen deprivation has become the main treatment for locally advanced disease. The approved prescription dose for these patients is 70 to 78 Gy delivered in $1.8-2$ Gy/fraction over 7-8 weeks with a pelvic lymph nodes (LNs) dose of 45-50 $\mathrm{Gy}^{2}$.

The escalation of the dose is now applicable using new radiotherapy techniques, such as intensitymodulated radiotherapy (IMRT), creating concave dose isodose lines around the target while protecting organs at risk (OARs) ${ }^{3}$. Compared to 3-dimensional conformal radiation therapy (3-D CRT), IMRT delivers a lower dose to the rectum and bladder with better coverage of the target volumes ${ }^{4}$. Comparison between volumetric- modulated arc therapy (VMAT) and IMRT in different studies showed that VMAT has better efficiency. Many trials confirmed that the rectum and bladder are better spared by VMAT than IMRT. VMAT treatment time is less than IMRT as fewer motor units (MUs) are needed to deliver the same dose distribution by single arc ${ }^{5-7}$.

At least $50 \%$ of the active bone marrow (BM) of the adults is present in the lumbar spine, sacral bone, ilium, ischium, pubic bones, and upper femur. Bone marrow is highly sensitive to radiation therapy due to its cytotoxic effect $^{8}$.

Androgen deprivation therapy (ADT) given concurrently with radiation therapy can increase its hematological toxicity. The hematological toxicity of ADT was not fully evaluated in prostate cancer clinical studies.

The blood lymphocytes are frequently assessed in radiobiological assays as it is easy to obtain and show high sensitivity to radiation ${ }^{9}$. The preclinical data and 
humans' limited information suggest that androgen hormones may have a role in regulating different hemopoietic cells. Limited data are available about the influence of ADT on immunity cells and platelets. In a trial evaluating 141 prostate cancer patients who received radiotherapy plus 4 months of combined androgen blockade, hemoglobin decreased by about $3.1 \mathrm{~g} / \mathrm{dl}$, and $81 \%$ of men developed hemoglobin drop of $>2 \mathrm{~g} / \mathrm{dl}{ }^{10}$. Thrombopoiesis is also regulated by androgens, as the androgen receptor is expressed on megakaryocytes and regulated by androgens ${ }^{11}$.

There is no specific recommendation regarding dose volume objectives for pelvic bone marrow (PBM) sparing to decrease the possibility of the hematological toxicities ${ }^{12}$. However, many studies revealed a correlation between the hematological toxicity and the PBM dosimetric parameters in anal and cervical carcinoma patients. In anal cancer patients, the suggested dose constraints to the lumbo-sacral spine were V10 $\leq$ $80 \%$. Keeping the mean dose of PBM $<22.5$ Gy and $<$ 25 Gy has been associated with $5 \%$ and $10 \%$ incidence of hematological toxicity, respectively ${ }^{13}$.

Studies that assessed cervical cancer suggested that PBM dose of $\mathrm{V} 10 \geq 90 \%$ had higher incidence of grade 2 hematological toxicity rates than PBM dose V10 < $90 \%{ }^{14}$.

We assume that both dose escalation and the combined use of ADT with radiotherapy can increase the hematological toxicity. Proper selection of the best technique in radiation therapy can decrease the PBM dose, and consequently decrease the hematological toxicity. In this study, we compared the dosimetric results of PBM preservation between dual arc VMAT (Rapid Arc, Varian model) and 7 fields IMRT in radical radiotherapy for prostate cancer who received pelvic LNs irradiation.

\section{METHODS}

\section{Study population}

26 patients with high-risk localized carcinoma of prostate were selected for this study and all were treated by radical radiotherapy including the pelvic LNs. Luteinising-hormone releasing hormone (LHRH) agonist was given concurrently with radiotherapy.

\section{Treatment planning}

Each patient was simulated in head first supine position. Computed tomographic images with a thickness of $3 \mathrm{~mm}$ were taken from upper abdomen to 5 $\mathrm{cm}$ below the ischial tuberosities with immobilization by a custom vacuum immobilization device, and with state of full bladder and empty rectum condition.

In each patient, two targets were defined, clinical target volume (CTV) including the prostate and seminal vesicles (CTV-PSV) and CTV including the pelvic LNs (CTV LN) common iliac below L5-S1, external iliac, internal iliac and obturator; with $7 \mathrm{~mm}$ around iliac vessels, carving out bowel, bladder and bone.

Based on standard Radiation Therapy Oncology Group (RTOG) guidelines, the OARs delineated were the rectum, urinary bladder, small intestine, bulb of the penis, femoral heads bilaterally and PBM. Bone marrow was delineated as the marrow cavity $2 \mathrm{~cm}$ above and below the planning target volume (PTV).

Two set of plans, one set of dual Arc VMAT and another set of 7-beams IMRT were generated and optimized with similar planning objectives to each patient. For all patients, the dose prescribed to the CTVPSV was 76 Gy in 38 fractions given over 7.5 weeks and the dose to CTV-LN was 54 Gy in 38 fractions given over 7.5 weeks. The PTV was created from the CTV with an additional margin of $5 \mathrm{~mm}$ in all direction.

The plans were analyzed according to the dosevolume histogram (DVH), and the coverage of the target volume V95\%. Calculation of all plans was done by 10 MV photon using anisotropic analytical algorithm (AAA).

The PBM dose was analyzed with different dose volume objectives since there is no exact dose volume objective of sparing level has been recommended. For assessment of PBM dose, V10, V20, V30, V40, V50 and mean dose were calculated for both VMAT and IMRT plans.

\section{Plan evaluation and acceptance}

All plans were evaluated based on DVH scoring values of PTVs and OARs. The PTV was evaluated using the conformity index (CI), in association with the homogeneity index (HI) and dose gradient index of the distribution of the dose. Conformity Index equals $\mathrm{V}_{\mathrm{RI}}$ / $\mathrm{TV}$, where " $\mathrm{V}_{\mathrm{RI}}$ " is reference isodose volume and "TV" is the target volume. Homogeneity Index equals $\mathrm{I}_{\max } / \mathrm{RI}$, where "I $I_{\max }$ " is maximum isodose in the target and "RI" is the reference isodose.

The statistical analysis was done with the use of SPSS (version 16.0.0, SPSS, Chicago, USA). P-value less than 0.05 was considered of significance statistically.

\section{RESULTS}

VMAT plans of most patients showed better DVH and CI than IMRT plans (table 1).

The mean dose of PTV 56 Gy for both techniques was satisfactory and comparable with better OARs (bladder and rectum) sparing using VAMT techniques.

The mean CI differed significantly between the VMAT and IMRT techniques, while the mean HI did not differ significantly between them (table 1).

The mean dose for PBM in the VMAT technique (21.7 Gy) was significantly less than that in the IMRT one $(25.8 \mathrm{~Gy})$ as shown in table 1 .

The axial, sagittal and coronal views of dose distribution for one of the study patients by both techniques are illustrated in figures 1 (IMRT) and 2 (VMAT).

Figure 3 shows the DVH for PBM of patient's plans. The mean values of V30 differed significantly between the IMRT and VMAT techniques $(\mathrm{p}<0.05)$. Similarly, the mean values of V40 differed significantly $(\mathrm{p}<0.05)$. 
Table 1: Comparison between volumetric-modulated arc therapy (VMAT) and intensity-modulated radiotherapy (IMRT) as regards conformity index (CI), homogeneity index (HI) and pelvic bone marrow (PBM) dose

\begin{tabular}{|c|c|c|c|c|c|c|}
\hline \multirow[t]{2}{*}{ Patient's No. } & \multicolumn{2}{|c|}{ CI } & \multicolumn{2}{|c|}{ HI } & \multicolumn{2}{|c|}{ PBM mean dose $(\mathrm{Gy})$} \\
\hline & IMRT & VMAT & IMRT & VMAT & IMRT & VMAT \\
\hline 1 & 1.08 & 1.05 & 0.072 & 0.084 & 26.65 & 24.46 \\
\hline 2 & 1.11 & 1.06 & 0.086 & 0.082 & 27.3 & 23.4 \\
\hline 3 & 1.06 & 1.035 & 0.092 & 0.12 & 26.4 & 22.6 \\
\hline 4 & 1.035 & 1.023 & 0.062 & 0.084 & 25.4 & 22 \\
\hline 5 & 1.092 & 1.053 & 0.102 & 0.1042 & 26.3 & 21.3 \\
\hline 6 & 1.085 & 1.046 & 0.091 & 0.093 & 24 & 21.7 \\
\hline 7 & 1.072 & 1.036 & 0.086 & 0.096 & 26.2 & 22.3 \\
\hline 8 & 1.12 & 1.082 & 0.078 & 0.085 & 22.3 & 19.6 \\
\hline 9 & 1.07 & 1.065 & 0.0692 & 0.078 & 27.3 & 22.1 \\
\hline 10 & 1.06 & 1.0387 & 0.058 & 0.068 & 26.3 & 22.8 \\
\hline 11 & 1.043 & 1.023 & 0.0762 & 0.087 & 28.4 & 21.3 \\
\hline 12 & 1.112 & 1.085 & 0.074 & 0.086 & 28.3 & 22.3 \\
\hline 13 & 1.034 & 1.03 & 0.086 & 0.092 & 25.38 & 19.6 \\
\hline 14 & 1.0914 & 1.062 & 0.113 & 0.063 & 26.4 & 22.1 \\
\hline 15 & 1.078 & 1.046 & 0.106 & 0.097 & 27.6 & 22.6 \\
\hline 16 & 1.046 & 1.035 & 0.089 & 0.1 & 26.3 & 21.78 \\
\hline 17 & 1.085 & 1.063 & 0.092 & 0.098 & 25.8 & 22.3 \\
\hline 18 & 1.054 & 1.043 & 0.093 & 0.103 & 24.6 & 21.3 \\
\hline 19 & 1.045 & 1.032 & 0.1 & 0.102 & 23.6 & 20.3 \\
\hline 20 & 1.062 & 1.053 & 0.098 & 0.0995 & 22.3 & 20.5 \\
\hline 21 & 1.132 & 1.057 & 0.086 & 0.092 & 28.6 & 23.5 \\
\hline 22 & 1.068 & 1.083 & 0.078 & 0.083 & 27.4 & 22.35 \\
\hline 23 & 1.022 & 1.018 & 0.076 & 0.0873 & 24.3 & 18.6 \\
\hline 24 & 1.083 & 1.067 & 0.089 & 0.0973 & 25.3 & 21.8 \\
\hline 25 & 1.138 & 1.069 & 0.068 & 0.0765 & 24.3 & 21.5 \\
\hline 26 & 1.123 & 1.052 & 00.102 & 0.098 & 25.1 & 22.3 \\
\hline Minimum & 1.022 & 1.018 & 0.058 & 0.063 & 22.3 & 18.6 \\
\hline Maximum & 1.138 & 1.085 & 0.113 & 0.765 & 28.6 & 24.46 \\
\hline Mean & 1.0769 & 1.0503 & 0.08548 & 0.09061 & 25.8396 & 21.7842 \\
\hline Standard deviation & 0.03156 & 0.01871 & 0.01381 & 0.0121 & 1.70948 & 1.28113 \\
\hline $\mathbf{t}$ & \multicolumn{2}{|c|}{3.708} & \multicolumn{2}{|c|}{-1.425} & \multicolumn{2}{|c|}{9.68} \\
\hline p value & \multicolumn{2}{|c|}{0.001} & \multicolumn{2}{|c|}{0.16} & \multicolumn{2}{|c|}{$<0.001$} \\
\hline
\end{tabular}

Bone marrow mean doses and different volumes start from V20, V30, V35 and V40 dose were $72.10 \pm$ $2.31,65.32 \pm 1.32,39.213 \pm 0.86$ and $24.8 \pm 1.3$ with IMRT and $18.6 \pm 1.56,13.42 \pm 1.22,11.18 \pm 2.3$ and $8.63 \pm 1.5$ with VMAT. The significant differences in PBM doses were in the range of 20Gy to 40Gy.

The other investigated BM volumes as V5, V10, V15, V45 and V50 showed no statistical significance between IMRT and VMAT.

The Dmax and mean dose for the rectum were significantly higher with IMRT (77.5 Gy and 42.1 Gy) than with VMAT (76.2 Gy and 38.7 Gy) ( $<<0.05)$. Similarly, the Dmax and mean dose for the bladder were significantly higher with IMRT (77.6 Gy and 45.3 Gy) than with VMAT (76.4 Gy and 42.5 Gy) (p<0.05).

For PTV 56 Gy Dmin, mean dose and Dmax were 55.4 Gy, 60 Gy and 78.2 Gy in IMRT plans respectively, while they were 57.3 Gy, 58.2 GY and 77.6 Gy in
VMAT plans respectively, with statistically significant difference in favor of VMAT $(\mathrm{p}<0.05)$.

For PTV 76 Gy Dmin, mean dose and Dmax were 70.8 Gy, 75.9 Gy and 80 Gy in IMRT plans respectively; while they were $71.8 \mathrm{~Gy}, 77.9 \mathrm{~Gy}$ and $78.6 \mathrm{~Gy}$ in VMAT plans respectively, with statistically significant difference in favor of VMAT $(\mathrm{p}<0.05)$.

\section{DISCUSSION}

Both radiation and ADT are myelosuppressive, and combined treatment can lead to significant hematological toxicity. The irradiated bone marrow volume and the received dose affect the extent if its injury as shown in clinical studies ${ }^{15}$

A large volume of active PBM is affected using the 3-D conformal technique, especially in the pelvic bones and lower vertebrae, but using IMRT technique can decrease the bone marrow that receives $20 \mathrm{~Gy}$ or higher. 


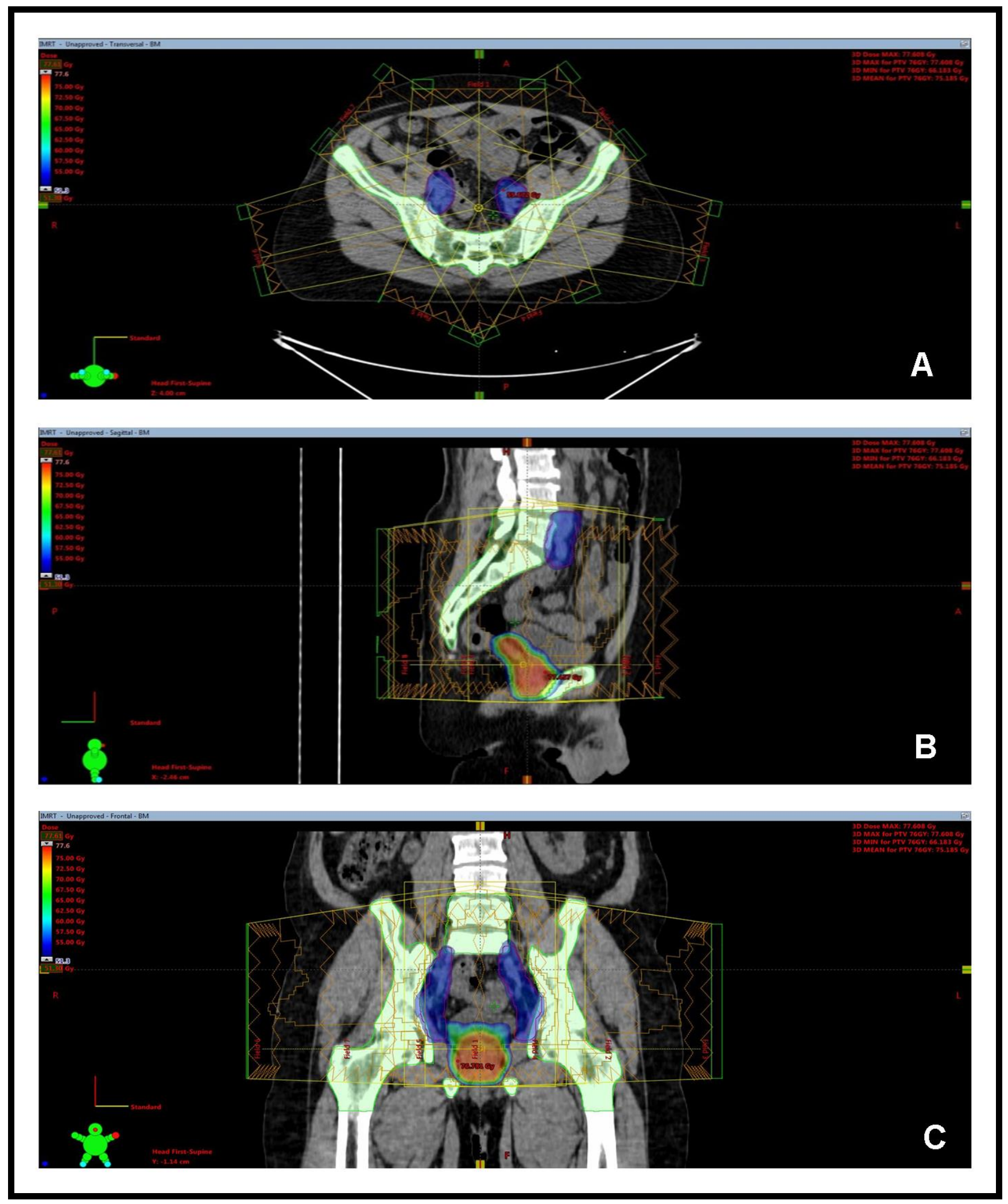

Figure 1: Dose distribution of intensity-modulated radiotherapy (IMRT) plan in one patient: A) Axial, B) Sagittal and C) Coronal views

The possibility of bone marrow sparing is limited by difficulties to avoid the large BM volume included in the radiation field ${ }^{16}$.

There is limited awareness of PBM delineation during the process of radiation, explained by the deficient data regarding the exact PBM tolerance doses and limited data that translate the DVH scores into definitive hematological changes. Based on many studies showing better OARs preservation (rectum and bladder) by VMAT when compared to IMRT 17, ${ }^{18}$, we hypothesized that VMAT may spare PBM better than IMRT with comparable PTV distribution. 


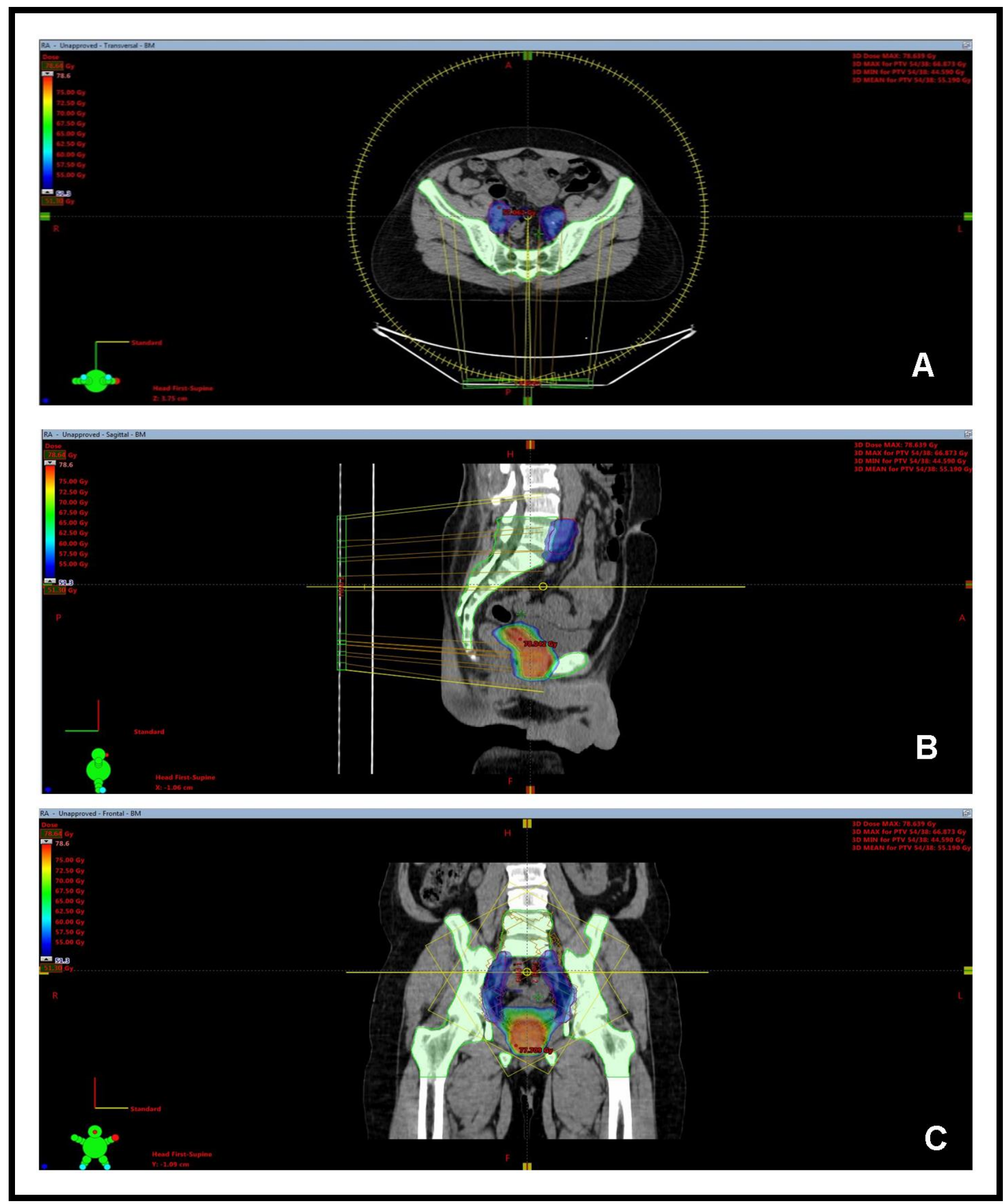

Figure 2: Dose distribution of volumetric-modulated arc therapy (VMAT) plan in one patient: A) Axial, B) Sagittal and C) Coronal views

In our study, Both VMAT and 7-fields IMRT showed satisfactory PTV 56 Gy and PTV 76 Gy coverage; however, VMAT showed higher PTV 56 conformality and homogeneity than IMRT.

Comparable results of PTV coverage were shown by Rao et al, who reported that more uniform plans were provided by VMAT than IMRT with more rapid treatment delivery and less MUs ${ }^{5}$.

Our results showed statistically significant better PBM sparing by VMAT than IMRT in terms of PBM mean dose, V20, V30 and V40. The Mean dose for PBM by VMAT technique was significantly less than IMRT. 


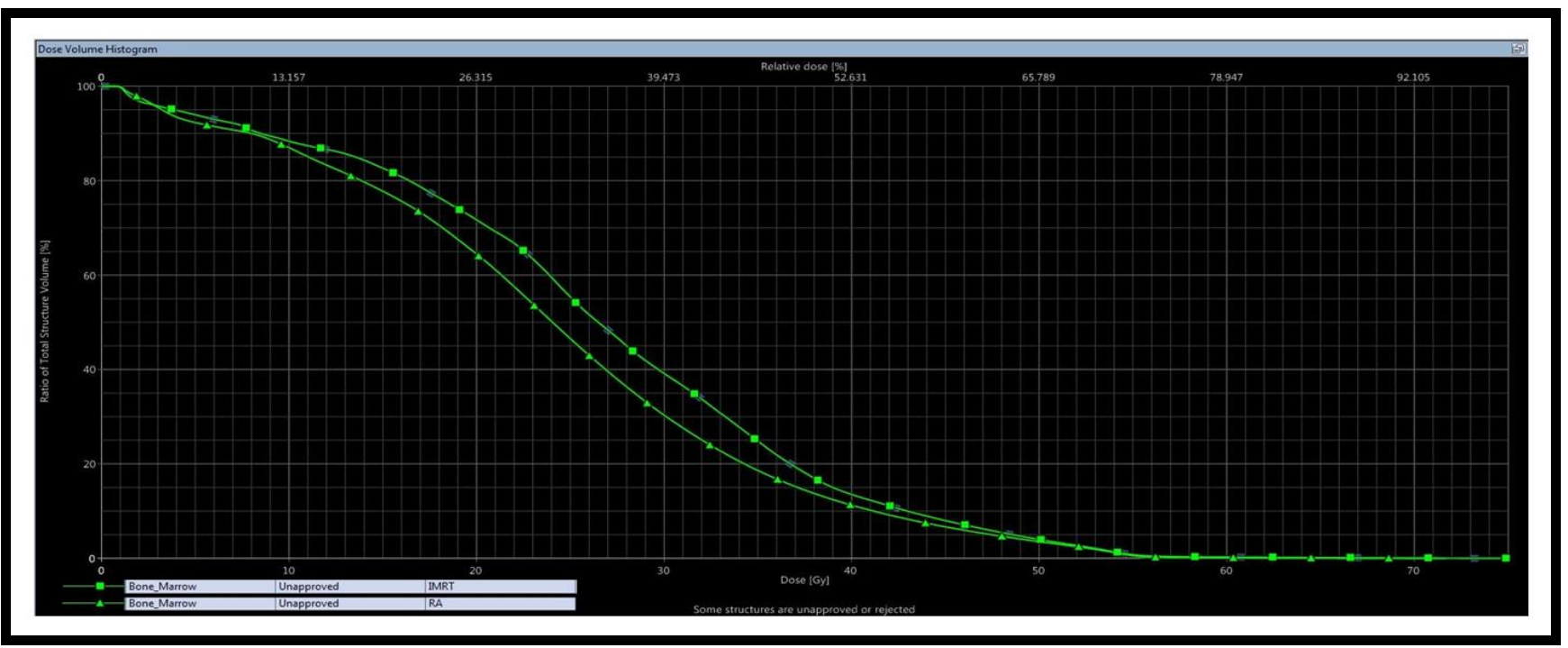

Figure 3: Dose-volume histogram (DVH) for pelvic bone marrow of patient's plans showing better sparing with volumetric-modulated arc therapy (VMAT) than intensity-modulated radiotherapy (IMRT)

Similar results were reported in another study, in which VMAT had better PBM preservation than IMRT in cervical cancer patients who received pelvic LNs irradiation, and the bone marrow mean and D40\% dose with VMAT and IMRT were $30.128 \pm 1.94$ and $34.399 \pm$ $2.09 ; 32.216 \pm 2.72$ and $37.397 \pm 2.87$ respectively ${ }^{19}$.

In this study, we observed that the difference between VMAT and IMRT was in the range including V20, V30 and V40 and also the mean PBM dose with a statistical significance. This observation raises the importance of the use of VMAT rather than IMRT, as according to RTOG 0418 phase II clinical trial, the higher the mean dose and more V40, the higher haematological toxicity of concurrent chemoradiotherapy in cervical carcinoma ${ }^{20}$.

Other studies showed that delayed recovery of BM volumes with possible irreversible damage occurs with $\mathrm{BM}$ doses more than 30 to $50 \mathrm{~Gy}^{8,9}$.

Our study showed that BM mean doses and different volumes start from V20, V30, V35 and V40 dose with IMRT and VMAT. This is comparable to another study that showed in which the mean of V10, V20, V30, and V40 of whole PBM were $80 \%, 36 \%, 77 \%$ and $36 \%$, respectively. However, in that trial V30, V40 of sacral bone and ischial bone were higher due to its closeness to the planning target volumes. The iliac bone V30 was relatively higher as sparing of the bowel loops lead to higher dose accumulation in the iliac bone ${ }^{21}$.

The matched results of many studies correlating the acute hematological toxicities, with the BM mean dose and the range between V20 to V40 may give specific suggestions regarding $\mathrm{BM}$ tolerance dose during radiotherapy planning in pelvic lymph node irradiation for prostatic carcinoma.

According to our study, we suggest that the use of VMAT can lead to decrease in hematological toxicity through the reduction of specific clinically important PBM dose volume levels.

Further clinical trials are needed to correlate these PBM doses with clinical acute BM toxicity, as more sparing of PBM can lead to less risk of hematological toxicity.

\section{Conclusion}

In high-risk prostate cancer patients receiving pelvic LNs irradiation, VMAT has better coverage of the target with better PBM sparing than IMRT. The toxicity of BM needs to be correlated with radiation dose and volume in future clinical trials. Increased awareness of PBM delineation and defining a clear BM tolerance can lead to decreased hematological toxicity.

\section{CONFLICT OF INTEREST}

The authors have no conflict of interest to declare

\section{REFERENCES}

1. Heidenreich A, Bellmunt J, Bolla M, et al. EAU guidelines on prostate cancer. Part 1: screening, diagnosis, and treatment of clinically localised disease. Eur Urol. 2011; 59(1): 61-71.

2. Bolla M, Collette L, Blank L, et al. Long-term results with immediate androgen suppression and external irradiation in patients with locally advanced prostate cancer (an EORTC study): a phase III randomised trial. Lancet. 2002; 360(9327): 103-106.

3. Martinez AA, Yan D, Lockman D, et al. Improvement in dose escalation using the process of adaptive radiotherapy combined with three-dimensional conformal or intensitymodulated beams for prostate cancer. Int J Radiat Oncol Biol Phys. 2001; 50(5): 1226-1234.

4. Luo C, Yang CC, Narayan S, et al. Use of benchmark dose-volume histograms for selection of the optimal technique between three-dimensional conformal radiation therapy and intensity-modulated radiation therapy in prostate cancer. Int J Radiat Oncol Biol Phys. 2006; 66(4): 1253-1262.

5. Rao M, Yang W, Chen F, et al. Comparison of Elekta VMAT with helical tomotherapy and fixed field IMRT: plan quality, delivery efficiency and accuracy. Med Phys 2010; 37(3): 1350-1359. 
6. Sze HC, Lee MC, Hung WM, Yau TK, Lee AW. RapidArc radiotherapy planning for prostate cancer: single-arc and double-arc techniques vs. intensitymodulated radiotherapy. Med Dosim. 2012; 37(1): 87-91.

7. Wolff D, Stieler F, Welzel G, et al. Volumetric modulated arc therapy (VMAT) vs. serial tomotherapy, step-andshoot IMRT and 3D-conformal RT for treatment of prostate cancer. Radiother Oncol. 2009; 93(2): 226-233.

8. Cao X, Wu X, Frassica D, et al. Irradiation induces bone injury by damaging bone marrow microenvironment for stem cells. Proc Natl Acad Sci U S A. 2011; 108(4): 16091614.

9. Grossmann M, Zajac JD. Hematological changes during androgen deprivation therapy. Asian J Androl. 2012; 14(2): 187-192.

10. Asbell SO, Leon SA, Tester WJ, Brereton HD, Ago CT, Rotman M. Development of anemia and recovery in prostate cancer patients treated with combined androgen blockade and radiotherapy. Prostate. 1996; 29(4): 243248.

11. Khetawat G, Faraday N, Nealen ML, et al. Human megakaryocytes and platelets contain the estrogen receptor $\beta$ and androgen receptor (AR): testosterone regulates AR expression. Blood. 2000; 95(7): 2289-2296.

12. Sini C, Fiorino C, Perna L, et al. Dose-volume effects for pelvic bone marrow in predicting hematological toxicity in prostate cancer radiotherapy with pelvic node irradiation. Radiother Oncol. 2016; 118(1):79-84.

13. Bazan JG, Luxton G, Mok EC, Koong AC, Chang DT. Normal tissue complication probability modeling of acute hematologic toxicity in patients treated with intensitymodulated radiation therapy for squamous cell carcinoma of the anal canal. Int J Radiat Oncol Biol Phys. 2012; 84(3): 700-706.

14. Mell LK, Kochanski JD, Roeske JC, et al. Dosimetric predictors of acute hematologic toxicity in cervical cancer patients treated with concurrent cisplatin and intensitymodulated pelvic radiotherapy. Int J Radiat Oncol Biol Phys. 2006; 66(5): 1356-1365.

15. Rose BS, Aydogan B, Liang Y, et al. Normal tissue complication probability modeling of acute hematologic toxicity in cervical cancer patients treated with chemoradiotherapy. Int J Radiat Oncol Biol Phys. 2011; 79(3): 800-807.

16. Liang $\mathrm{Y}$, Messer $\mathrm{K}$, Rose BS, et al. Impact of bone marrow radiation dose on acute hematologic toxicity in cervical cancer: Principal component analysis on high dimensional data. Int J Radiat Oncol Biol Phys. 2010; 78(3): 912-919.

17. Hardcastle N, Tomé WA, Foo K, Miller A, Carolan M, Metcalfe P. Comparison of prostate IMRT and VMAT biologically optimised treatment plans. Med Dosim. 2011; 36(3): 292-298

18. Kopp RW, Duff M, Catalfamo F, Shah D, Rajecki M, Ahmad K. VMAT vs. 7-field-IMRT: Assessing the dosimetric parameters of prostate cancer treatment with a 292-patient sample. Med Dosim. 2011; 36(4): 365-372.

19. Deng X, Han C, Chen S, et al. Dosimetric benefits of intensity-modulated radiotherapy and volumetricmodulated arc therapy in the treatment of postoperative cervical cancer patients. J Appl Clin Med Phys. 2017; 18(1): 25-31.

20. Klopp AH, Moughan J, Portelance L, et al. Hematologic toxicity in RTOG 0418: a phase 2 study of postoperative IMRT for gynecologic cancer. Int J Radiat Oncol Biol Phys. 2013; 86(1): 83-90.

21. Lewis S, Chopra S, Naga P, et al. Acute hematological toxicity during post-operative bowel sparing imageguided intensity modulated radiation with concurrent cisplatin. Br J Radiol. 2018; 91(1092): 20180005. 